\title{
Generation of a Purely Single Transverse Mode Vortex Beam from a He-Ne Laser Cavity with a Spot-Defect Mirror
}

\author{
Ken Kano, Yuichi Kozawa, and Shunichi Sato \\ Institute of Multidisciplinary Research for Advanced Materials, Tohoku University, Katahira 2-1-1, Aoba-ku, Sendai 980-8577, Japan
}

Correspondence should be addressed to Ken Kano, kkano@mail.tagen.tohoku.ac.jp

Received 7 June 2011; Accepted 3 August 2011

Academic Editor: Paramasivam Senthilkumaran

Copyright () 2012 Ken Kano et al. This is an open access article distributed under the Creative Commons Attribution License, which permits unrestricted use, distribution, and reproduction in any medium, provided the original work is properly cited.

Spot-defect mirrors were fabricated by focusing laser pulses on the surface of conventional dielectric mirrors. These mirrors were used as rear mirrors of a He-Ne laser cavity for generating a vortex beam. The intensity distribution of the beam generated from the cavity with a spot diameter of $50 \mu \mathrm{m}$ was in excellent agreement with theory. Comprehensive analysis of the intensity distribution, the beam quality factor, and the interference pattern revealed that the beam obtained was a purely single transverse mode $\mathrm{LG}_{01}$ beam.

\section{Introduction}

Laguerre-Gaussian (LG) beams have been highlighted due to its dominant feature of carrying optical angular momenta in singular optics [1]. They have brought about many applications such as optical spanners for driving micromachines, quantum information processing [2], superresolution microscopy [3], and microneedle fabrication [4]. To enhance the validity of these applications, the generation of a stable and high-quality LG mode beam is inevitable.

LG beams can be produced by extracavity methods such as a mode converter composed of a pair of cylindrical lenses [5], computer-generated holograms [6-8], diffractive optics [9], an adaptive helical mirror [10], and a spatial light modulator [11]. However, the misalignment and the imperfection of the optical elements gave rise to the degradation of LG beams. By contrast, direct generation from a laser cavity is expected to improve the beam quality due to an inherent feedback effect in the cavity. Although many attempts have been reported, for example, a ring-shaped beam pumping [12], the thermal lensing effect in a diode-side-pumped bounce laser cavity [13], the insertion of a spiral phase plate [14], and the use of a circular absorber [15-17], most of them have merely reported the generation of the LG beam without a detailed evaluation of the beam purity and quality. Recently, we have demonstrated the generation of LG beams from a $\mathrm{Nd}$ :yttrium aluminum garnet (YAG) laser cavity using a spot defect mirror [18]. This method is simple, robust, and applicable to many laser systems. In addition, a Gaussian mode will be strongly suppressed by properly choosing a diameter of the defect leading to the generation of a pure single transverse mode.

In this paper, we demonstrate the generation of a purely single transverse mode LG beam from a He-Ne laser cavity with a spot defect mirror. For mirrors with different spot diameters, comprehensive analysis was performed for the intensity distribution, the beam quality factor, and the observed interference pattern. The generation of a purely single transverse mode $\mathrm{LG}_{01}$ beam was verified.

\section{Experimental Setup}

Spot defect mirrors were fabricated by focusing Ti:Sapphire laser pulses near the surface of a conventional dielectric multilayer mirror. A circular part of the multilayer coating on the mirror surface was ablated to create a spot defect with low reflectivity. By changing the distance between the mirror surface and the focus, spot defects with different diameter $d$, of 13,20 , and $50 \mu \mathrm{m}$ were fabricated. Figure 1 (a) shows a photograph of the fabricated spot defect mirror. A small spot defect exists in the center of the mirror though it is hardly recognized in the photograph. The inset of Figure 1(a) is a reflective optical microscope image of the defect on the mirror surface fabricated by pulsed laser irradiation. The 


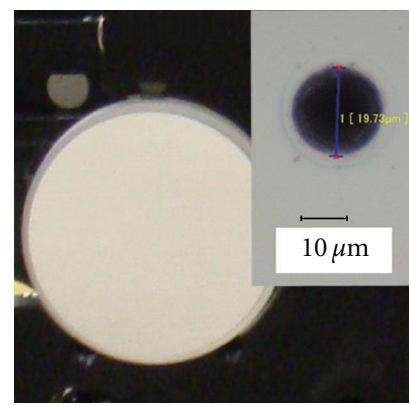

(a)

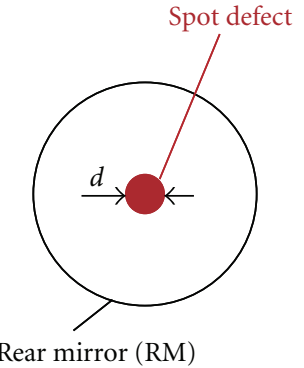

(b)

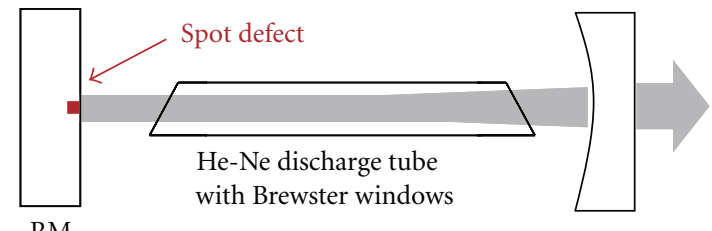

(flat)
OC $(R=98.9 \%)$ $(\mathrm{ROC}=600 \mathrm{~mm})$

(c)

FIGURE 1: (a) A photograph of the fabricated spot defect mirror. The inset is a reflective optical microscope image of the mirror surface with a spot defect diameter of $20 \mu \mathrm{m}$. Schematics of the spot defect mirror (b) and the He-Ne laser cavity (c).

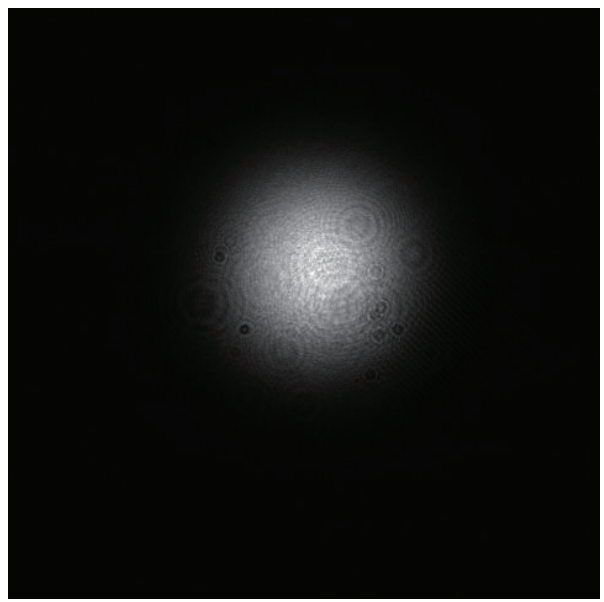

(a)

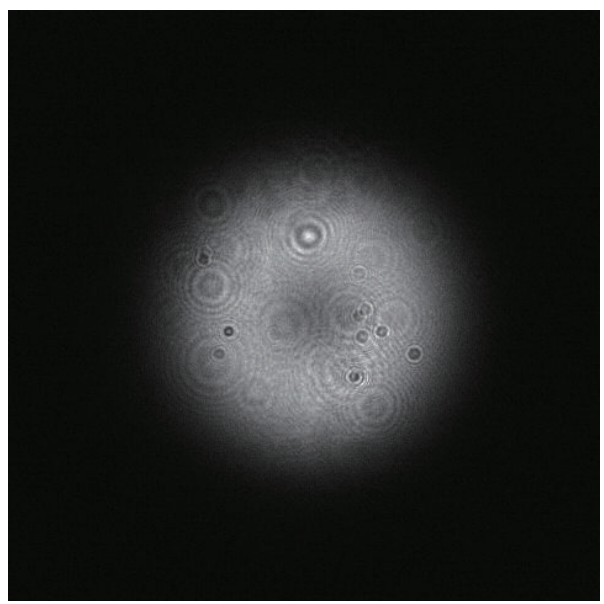

(c)

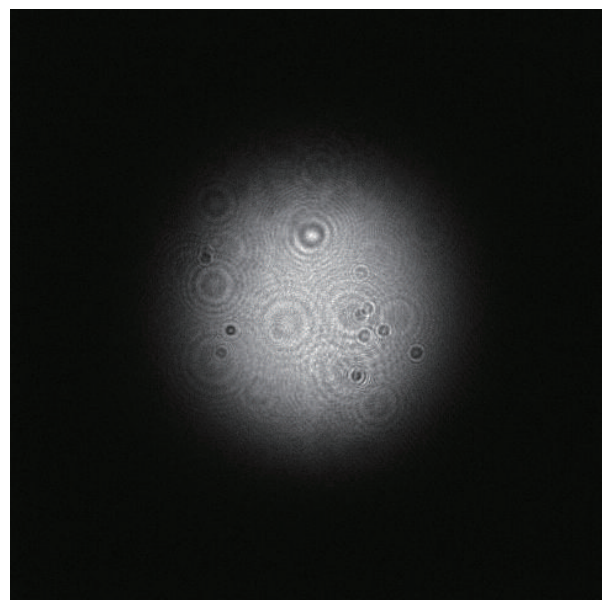

(b)

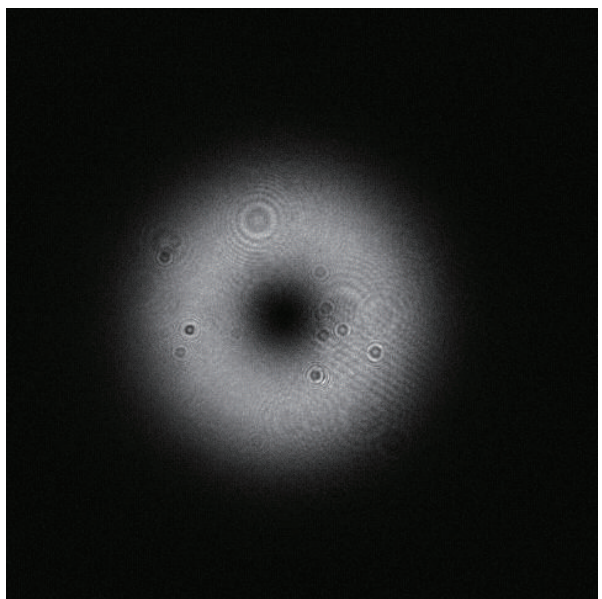

(d)

FIGURE 2: Intensity distributions of the generated beams for different spot defect diameters $d$ of (a) $0 \mu \mathrm{m}$, (b) $13 \mu \mathrm{m},(\mathrm{c}) 20 \mu \mathrm{m}$, and (d) $50 \mu \mathrm{m}$.

spot is a nearly perfect circle with a diameter of $20 \mu \mathrm{m}$. The shape of other spot defects has similar circularity.

Figure 1(b) illustrates a schematic of the spot defect mirror, which was used as a rear mirror (RM) of a He-Ne laser cavity as shown in Figure 1(c). The RM was mounted on an $X-Y$ mechanical stage. The cavity length and the distance between the RM and a discharge tube (length: $192 \mathrm{~mm}$; inner diameter: $3.7 \mathrm{~mm}$; terminated by Brewster windows) were $300 \mathrm{~mm}$ and $78 \mathrm{~mm}$, respectively. The radius of curvature ROC and the reflectance $R$ of an output coupler (OC) 


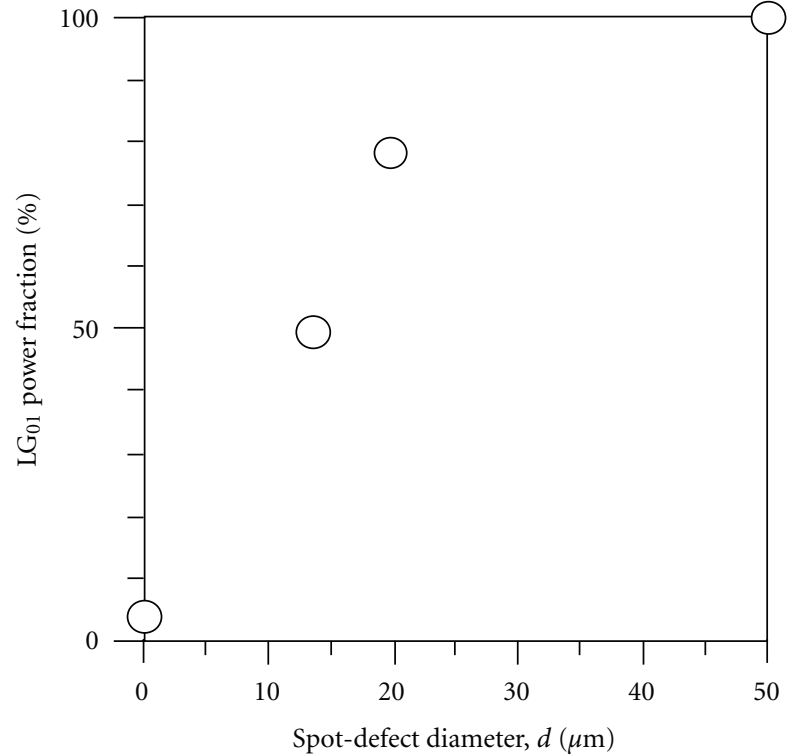

FIGURE 3: $\mathrm{LG}_{01}$ power fractions for different spot defect diameters $d$ (open circles).

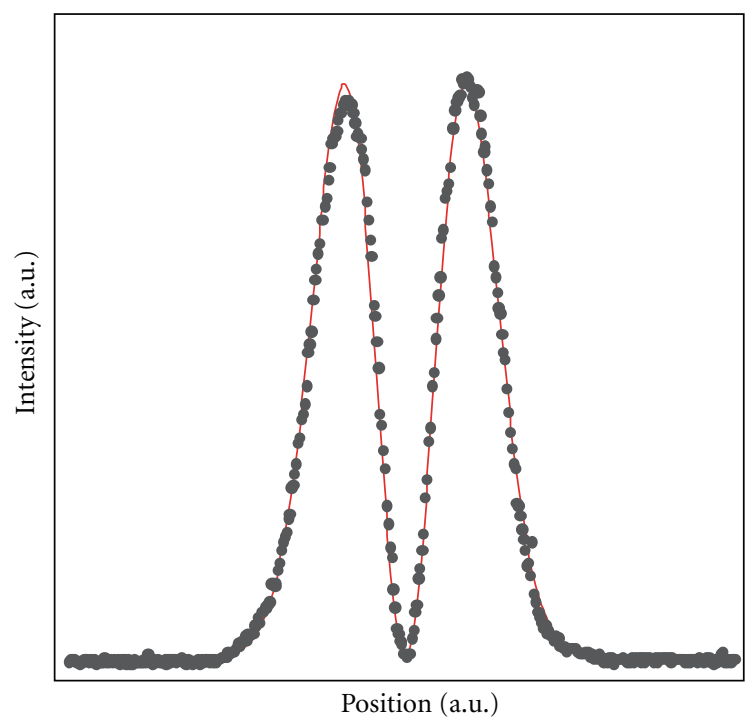

Figure 4: A cross-section of the intensity distribution of the beam for $d=50 \mu \mathrm{m}$. Dotted lines and a red solid curve indicate the measured data and fitting result, respectively.

were $600 \mathrm{~mm}$ and 98.9\%, respectively. Intensity distributions of the laser beams were recorded by a beam profiler. Polarization was verified from the intensity distributions after passing through a linear polarizer. Optical power was measured by an optical power meter.

To align the spot defect just on the cavity axis, the position of the RM was adjusted in the following procedure. First, a normal laser oscillation without a spot defect was achieved by putting the spot defect off the cavity axis. Then, the mirror was carefully moved to make the center of the spot defect coincide with the cavity axis. When the center of the spot defect is exactly on the cavity axis, the generated beam pattern drastically changed. This procedure was readily conducted by manually translating the $X-Y$ mechanical stage.

Note that the spot defect located on the beam axis is expected to readily generate a doughnut-like beam [18]. In addition, the Brewster plate inside a He-Ne laser cavity ensures the linear polarization. Under this condition, the most probable beam is a linearly polarized LG beam with an optical vortex.

\section{Results and Discussion}

Figure 2 shows the intensity distributions of the generated beams for different $d$. Note that all the beams were linearly polarized in the horizontal direction owing to the Brewster windows of the discharge tube. Without a spot defect $(d=0 \mu \mathrm{m})$, a Gaussian beam was obtained as shown in Figure 2(a). With increasing $d$, the intensity distribution changed to doughnut shape as shown in Figures 2(b)-2(d) due to the stronger suppression of a Gaussian mode by a larger spot defect. The output power was $1.0 \mathrm{~mW}$ except for the case without a spot defect $(1.5 \mathrm{~mW})$.

To evaluate the transverse mode purity of the generated laser beam, two-dimensional fitting analysis to the recorded intensity distribution was performed. A superposition of theoretical intensity distributions of a Gaussian $\left[I_{00}(x, y)\right]$ and $\mathrm{LG}_{01}$ mode $\left[I_{01}(x, y)\right]$ was adopted as a fitting function $I(x, y)$ and is given by

$$
I(x, y)=C_{00} I_{00}(x, y)+C_{01} I_{01}(x, y)+\mathrm{BG},
$$

where $C_{00}$ and $C_{01}$ represent power fractions of a Gaussian and a $\mathrm{LG}_{01}$ mode beam, respectively, and BG is background intensity. An intensity distribution of a $L_{p m}$ mode beam is the square of the absolute value of its complex amplitude, which is expressed in the cylindrical coordinate $(r, \theta, z)$ as

$$
\begin{aligned}
u_{p m}(r, \theta, z)= & \sqrt{\frac{2 p !}{\pi(|m|+p) !}} \frac{1}{w(z)} \times\left(\sqrt{2} \frac{r}{w(z)}\right)^{|m|} \\
& \times L_{p}^{|m|}\left(\frac{2 r^{2}}{w^{2}(z)}\right) \exp \left(-\frac{r^{2}}{w^{2}(z)}\right) \\
& \times \exp \left(-\frac{i k r^{2}}{2 R(z)}-i k z-i m \theta\right. \\
& +i(2 p+|m|+1) \Psi(\mathrm{z})),
\end{aligned}
$$

where $p$ and $m$ are the radial and azimuthal mode indices, respectively. $k$ is the wave number and $w(z)$ is the Gaussian beam width defined as $w(z)=w_{0} \sqrt{1+\left(z / z_{R}\right)^{2}}$, with the minimum beam radius $w_{0}$ at $z=0$ and the Rayleigh length $z_{R}=k w_{0}^{2} / 2 . R(z)=z+\left(z_{R}^{2} / z\right)$ is the wave-front radius of curvature, $(2 p+|m|+1) \Psi(z)$ is the Gouy phase shift with $\Psi(z)=\arctan \left(z / z_{R}\right)$, and $L_{p}^{|m|}$ is the generalized Laguerre polynomial. Note that fitting analysis was performed in the Cartesian coordinate system. Complex amplitude $u_{p m}(r$, $\theta)$ was transformed into $u_{p m}(x, y)$ by using following relationships $x=r \cos (\theta)$ and $y=r \sin (\theta)$. By the twodimensional fitting, the parameters including $C_{00}, C_{01}, \mathrm{BG}$, 


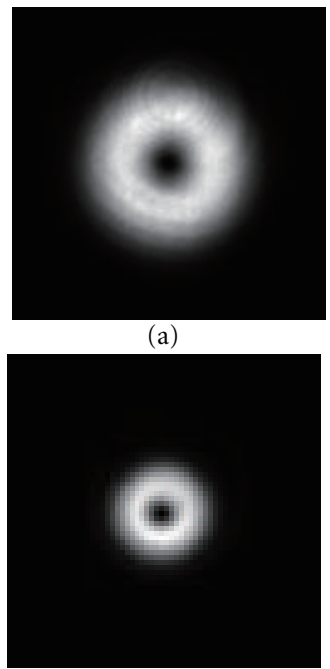

(b)

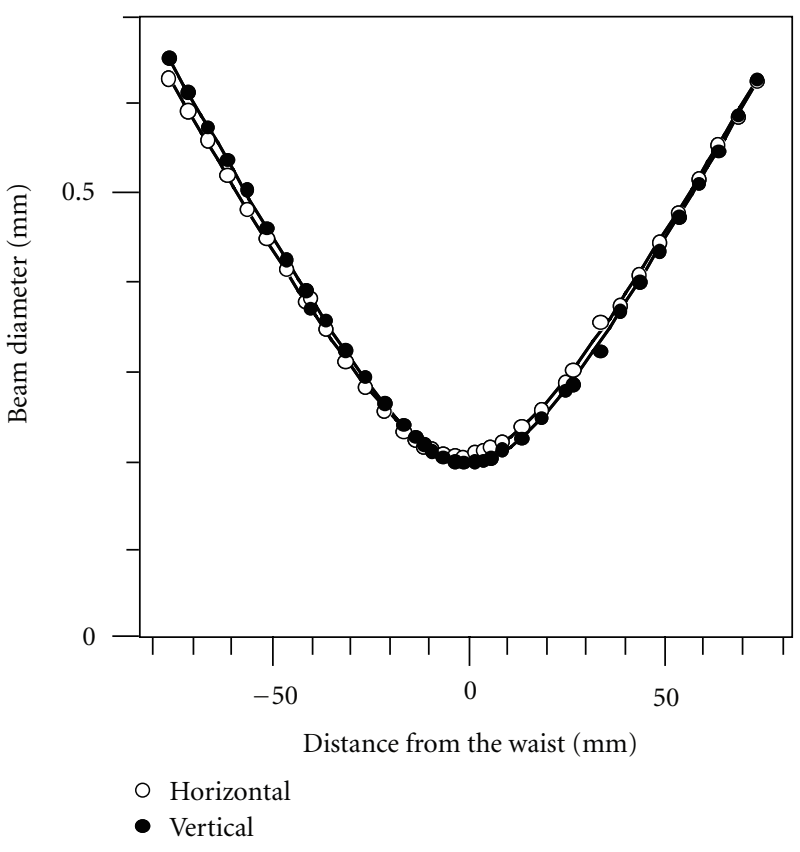

(c)

Figure 5: Recorded intensity distributions (a) before the waist by 4 times of Rayleigh length and (b) at the waist, respectively. (c) The variations of the beam diameters as a function of the distance from the waist. Open and filled circles indicate the horizontal and vertical beam widths, respectively. Fitting curves are depicted by solid lines.

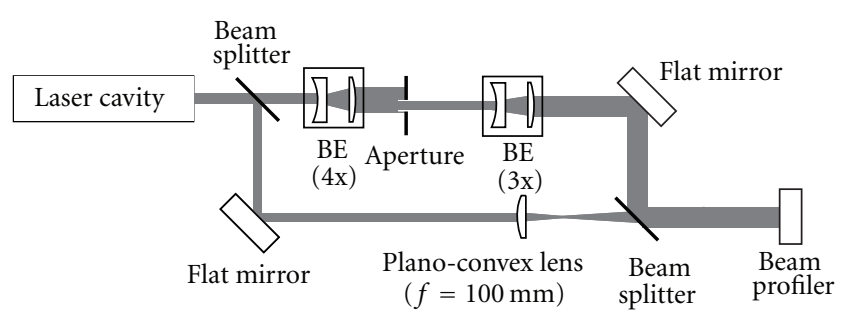

Figure 6: Optical schematic of the Mach-Zehnder interferometer.

center position, and the beam radius were determined. Twodimensional analysis enables direct and rigorous comparison between the theoretical profiles and the observed patterns avoiding ambiguity caused by an intentional selection of an intensity profile for the one-dimensional fitting. Figure 3 shows the $\mathrm{LG}_{01}$ power fraction estimated by $C_{01} /\left(C_{00}+C_{01}\right)$ as a function of $d$. The $\mathrm{LG}_{01}$ power fraction was about $4 \%$ for $d$ $=0 \mu \mathrm{m}$. With increasing $d$, the $\mathrm{LG}_{01}$ power fraction increased and reached $99.99 \%$ for $d=50 \mu \mathrm{m}$. Note that the selection of proper size of the spot defect is important to eliminate a Gaussian mode component.

Figure 4 shows a typical intensity distribution profile for $d=50 \mu \mathrm{m}$ (dotted lines) and the fitting curve of a theoretical $\mathrm{LG}_{01}$ mode (red solid line). The profile was in excellent agreement with that of a $L_{01}$ mode beam. Note that the intensity on the beam axis was almost zero meaning that a Gaussian mode component was negligibly small. A small difference of the peak height between two lobes may arise from the residual misalignment of the laser cavity mirrors.
Theoretical values of beam quality factor $M^{2}$ are 1 and 2 for Gaussian and $\mathrm{LG}_{01}$ mode beams, respectively. The value is between 1 and 2 when a laser beam contains both Gaussian and $\mathrm{LG}_{01}$ components. The $M^{2}$ of the beam generated for $d=50 \mu \mathrm{m}$ was measured by using a planoconvex lens with a focal length $f$ of $250 \mathrm{~mm}$. Recorded images of the beam crosssection before the waist by 4 times of Rayleigh length and at the waist are shown in Figures 5(a) and 5(b), respectively. The doughnut-shaped pattern was maintained throughout the focusing suggesting that the beam is an eigen mode. Figure 5(c) shows the variations of the beam diameter along the horizontal (open circle) and vertical (filled circle) axes as a function of the distance from the waist. Fitting curves are depicted by solid curves. Both of the beam quality factors for the horizontal and vertical axes were determined to 2.0. This value is exactly the same with the theoretical value for a $L_{01}$ mode beam.

The phase structure (spiral phase front) of the beam generated for $d=50 \mu \mathrm{m}$ was confirmed by measuring an interference pattern with a reference plane wave using a Mach-Zehnder interferometer. Figure 6 shows a schematic of a Mach-Zehnder interferometer. The output beam from the He-Ne laser cavity with a spot defect mirror was divided into two beams by a beam splitter. The upper beam shown in Figure 6 was expanded by a beam expander (BE) with a pair of lenses, clipping a small portion of the beam through a small aperture and then expanded again by a $\mathrm{BE}$ to generate a reference plane wave. At the camera position, the reference beam had a Gaussian-like intensity distribution with some fringes due to diffraction by the aperture. In the lower arm, 


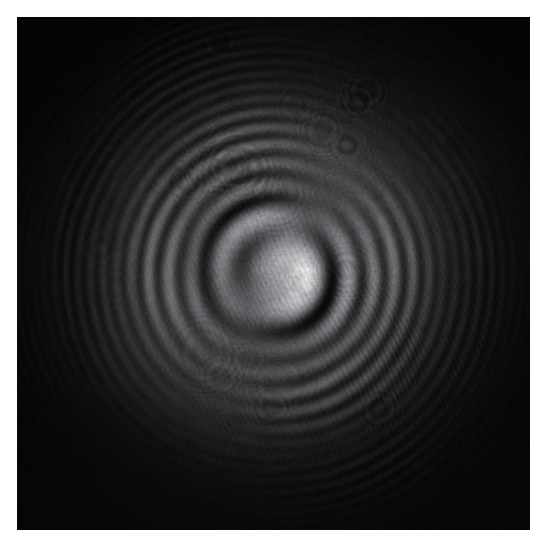

(a)

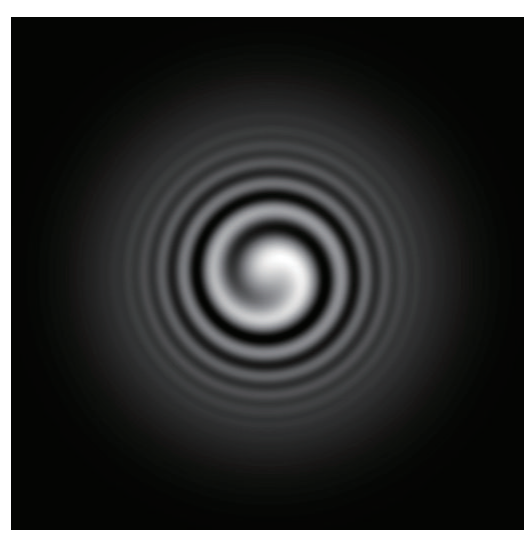

(b)

Figure 7: (a) Measured interference pattern between the beam generated for $d=50 \mu \mathrm{m}$ and a plane reference wave. (b) Calculated interference pattern between corresponding beams to (a).

the wave front of the signal wave was converted to a spherical one by a planoconvex lens with $f=100 \mathrm{~mm}$. Figure $7(\mathrm{a})$ shows the observed interference pattern. Figure 7 (b) depicts a calculated interference pattern of an ideal spherical $\mathrm{LG}_{01}$ mode beam with a collimated Gaussian beam. The interference fringe becomes obscure beyond the first three rings in Figure 7 (b) due to the inherent mismatch of intensity distributions between a doughnut-shaped $\mathrm{LG}_{01}$ mode beam and a Gaussian beam. The comparison of Figures 7(a) and 7 (b) strongly suggests that the beam generated for $d=50 \mu \mathrm{m}$ is a purely single transverse $\mathrm{LG}_{01}$ mode beam with the phase dependence of $\exp (-i \theta)$, namely, the topological charge of 1 . The imperfection of the contrast of the interference pattern and the continuity of the spiral in Figure $7(a)$ compared with Figure 7(b) may be attributed to the distortion of the reference wave generated by clipping a small portion of the $\mathrm{LG}_{01}$ mode beam through many optical elements as shown in Figure 6.

The comparison of power fraction of $\mathrm{LG}_{01}$ mode to higher order modes may be suitable to discuss the mode purity of the beam generated in this experiment. The power fractions of $L_{01}$ mode generated by using a computer-generated hologram and diffractive optics have been reported to be $93 \%$ [8] and 92.9\% [9], respectively. Following Kennedy et al. [9], the power fraction in our case was calculated to be $96.5 \%$, which is much higher than those ever reported indicating that the mode purity of the $\mathrm{LG}_{01}$ mode obtained in this experiment was quite high. In this calculation, higher order modes up to 6th order were considered.

The generated $\mathrm{LG}_{01}$ mode was quite stable. The doughnut-shaped intensity pattern was maintained over several hours. Mechanical vibrations produced in a normal laboratory resulted in no notable effects in our experimental condition in which the laser cavity was constructed on a vibration isolated table. The helicity of the $\mathrm{LG}_{01}$ beam mode was robust against a slight change of the alignment of the cavity mirrors. In addition, the $\mathrm{LG}_{01}$ beam with the same helicity was reproducibly generated after switching off and on the power source of the discharge or inserting an obstacle into the cavity to stop the oscillation. The stability and reproducibility observed here have been reported for a $\mathrm{Nd}$ : YAG laser cavity with a spot defect mirror [18].

A beam radius $w$ on the RM was estimated to be $0.246 \mathrm{~mm}$ by the $A B C D$ matrix formalism. The ratio of the spot defect diameter $(d=50 \mu \mathrm{m})$ to the beam diameter $[=d /(2 w)]$ was 0.102 in contrast to 0.207 observed for a $\mathrm{Nd}$ : YAG laser [18]. This difference can be attributed to the smaller gain of a He-Ne laser compared to a Nd: YAG laser.

\section{Conclusion}

Spot defect mirrors with the diameters $d$ of 13, 20, and $50 \mu \mathrm{m}$ were fabricated by focusing laser pulses on the surface of commercially available dielectric mirrors used as rear mirrors of a He-Ne laser cavity. With increasing $d$, the intensity distribution of the generated beam changed from a Gaussian beam to a doughnut-shaped pattern. The intensity distribution obtained for $d=50 \mu \mathrm{m}$ was in excellent agreement with that of a $\mathrm{LG}_{01}$ mode beam. The beam quality factor also agreed well with the theoretical value of a $\mathrm{LG}_{01}$ beam mode. The spiral interference pattern indicates that the generated beam had an $\exp (-i \theta)$ phase-front structure. By the careful analysis of the intensity distributions, the value of a beam quality factor, and observed interference pattern, the beam was verified to be a purely single transverse $\mathrm{LG}_{01}$ mode beam.

\section{References}

[1] L. Allen, S. M. Barnett, and M. J. Padgett, Optical Angular Momentum, Institute of Physics, Bristol, UK, 2003.

[2] S. Franke-Arnold, L. Allen, and M. Padgett, "Advances in optical angular momentum," Laser and Photonics Reviews, vol. 2, no. 4, pp. 299-313, 2008.

[3] E. Rittweger, K. Y. Han, S. E. Irvine, C. Eggeling, and S. W. Hell, "STED microscopy reveals crystal colour centres with nanometric resolution," Nature Photonics, vol. 3, no. 3, pp. 144-147, 2009. 
[4] T. Omatsu, K. Chujo, K. Miyamoto et al., "Metal microneedle fabrication using twisted light with spin," Optics Express, vol. 18, no. 17, pp. 17967-17973, 2010.

[5] M. W. Beijersbergen, L. Allen, H. E. L. O. van der Veen, and J. P. Woerdman, "Astigmatic laser mode converters and transfer of orbital angular momentum," Optics Communications, vol. 96, no. 1-3, pp. 123-132, 1993.

[6] N. R. Heckenberg, R. McDuff, C. P. Smith, and A. G. White, "Generation of optical phase singularities by computergenerated holograms," Optics Letters, vol. 17, no. 3, pp. 221223, 1992

[7] J. Arlt, K. Dholakia, L. Allen, and M. J. Padgett, "The production of multiringed Laguerre-Gaussian modes by computergenerated holograms," Journal of Modern Optics, vol. 45, no. 6, pp. 1231-1237, 1998.

[8] M. A. Clifford, J. Arlt, J. Courtial, and K. Dholakia, "Highorder Laguerre-Gaussian laser modes for studies of cold atoms," Optics Communications, vol. 156, no. 4-6, pp. 300306, 1998.

[9] S. A. Kennedy, M. J. Szabo, H. Teslow, J. Z. Porterfield, and E. R. I. Abraham, "Creation of Laguerre-Gaussian laser modes using diffractive optics," Physical Review A, vol. 66, no. 4, Article ID 43801, pp. 43801/1-43801/5, 2002.

[10] D. P. Ghai, "Generation of optical vortices with an adaptive helical mirror," Applied Optics, vol. 50, no. 10, pp. 1374-1381, 2011.

[11] N. Matsumoto, T. Ando, T. Inoue, Y. Ohtake, N. Fukuchi, and T. Hara, "Generation of high-quality higher-order LaguerreGaussian beams using liquid-crystal-on-silicon spatial light modulators," Journal of the Optical Society of America A, vol. 25, no. 7, pp. 1642-1651, 2008.

[12] Y. F. Chen and Y. P. Lan, "Dynamics of the Laguerre Gaussian $\mathrm{TEM}_{0, l}^{*}$ mode in a solid-state laser," Physical Review A, vol. 63, no. 6, Article ID 063807, pp. 063807/1-063807/9, 2001.

[13] S. P. Chard, P. C. Shardlow, and M. J. Damzen, "Highpower non-astigmatic $\mathrm{TEM}_{00}$ and vortex mode generation in a compact bounce laser design," Applied Physics B, vol. 97, no. 2, pp. 275-280, 2009.

[14] R. Oron, Y. Danziger, N. Davidson, A. A. Friesem, and E. Hasman, "Laser mode discrimination with intra-cavity spiral phase elements," Optics Communications, vol. 169, no. 1-6, pp. 115-121, 1999.

[15] C. Tamm, "Frequency locking of two transverse optical modes of a laser," Physical Review A, vol. 38, no. 11, pp. 5960-5963, 1988.

[16] L. E. Grin, P. V. Korolenko, and N. N. Fedotov, "Laser beams with a helical wavefront structure," Optics and Spectroscopy, vol. 73, no. 5, pp. 604-605, 1992.

[17] M. Harris, C. A. Hill, and J. M. Vaughan, "Optical helices and spiral interference fringes," Optics Communications, vol. 106, no. 4-6, pp. 161-166, 1994.

[18] A. Ito, Y. Kozawa, and S. Sato, "Generation of hollow scalar and vector beams using a spot-defect mirror," Journal of the Optical Society of America A, vol. 27, no. 9, pp. 2072-2077, 2010. 

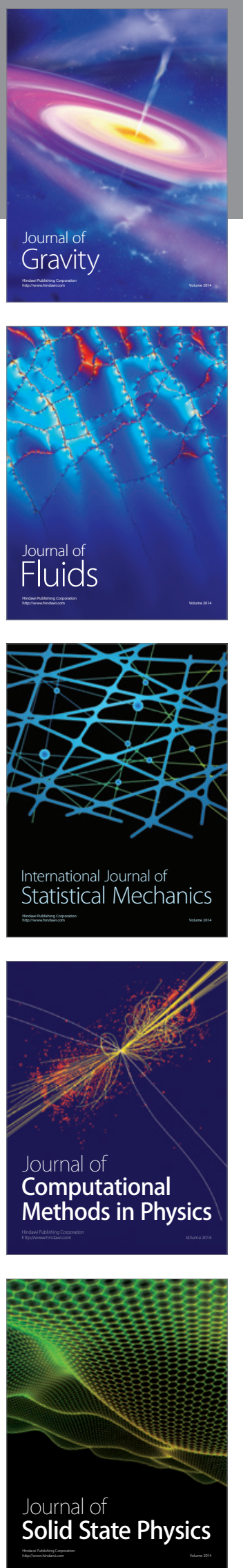

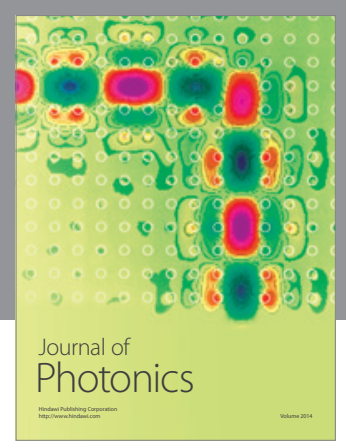

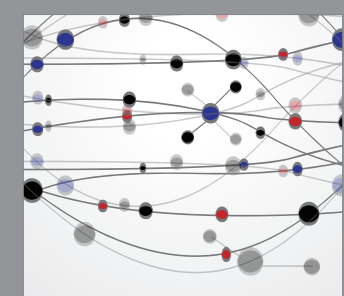

The Scientific World Journal
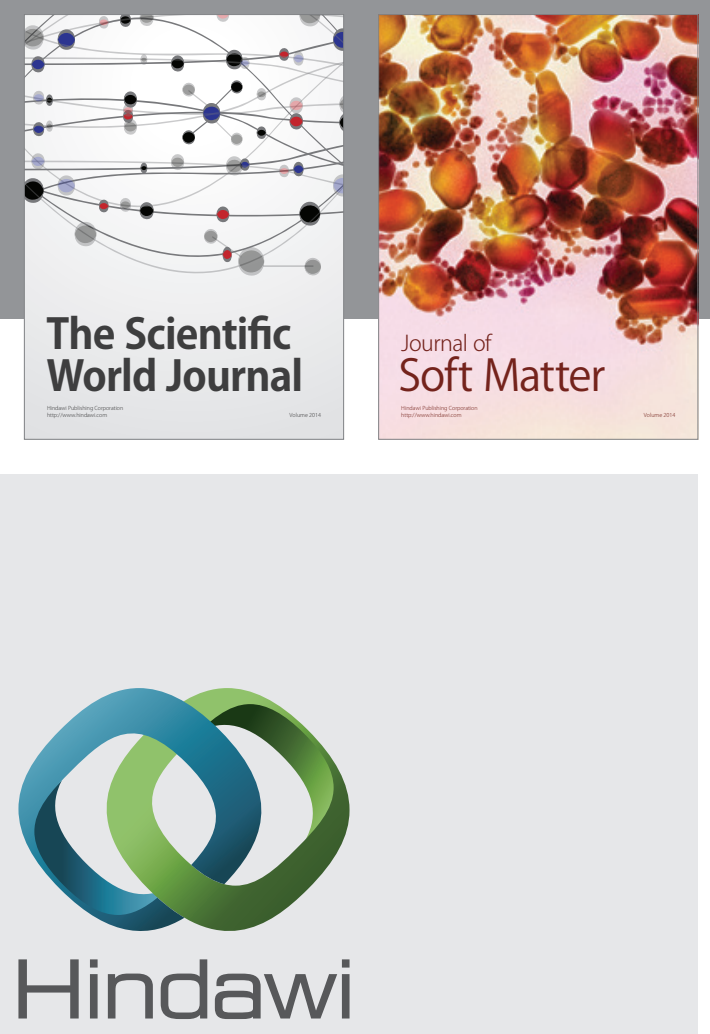

Submit your manuscripts at

http://www.hindawi.com
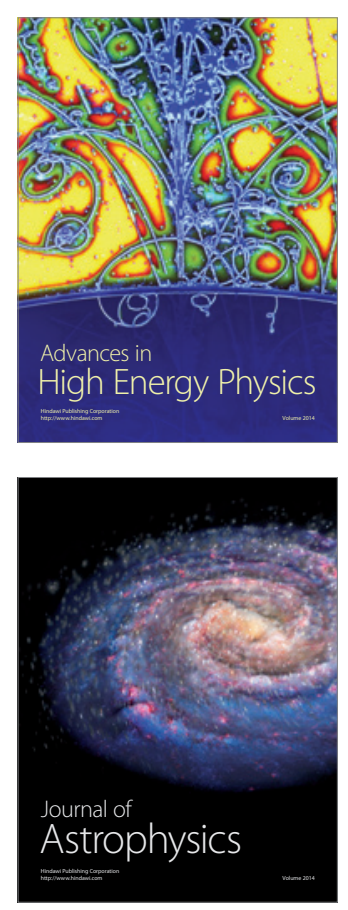
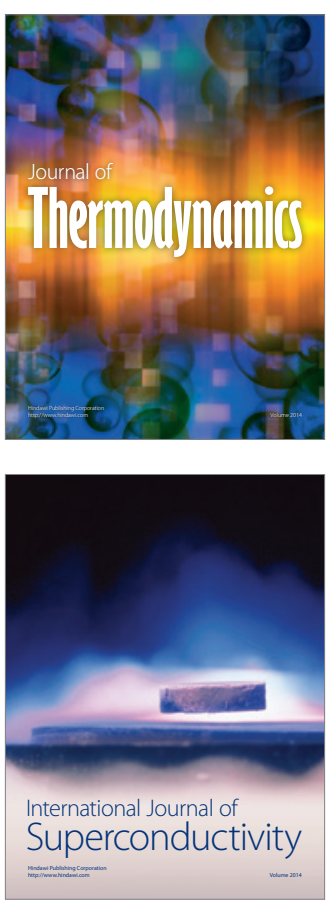
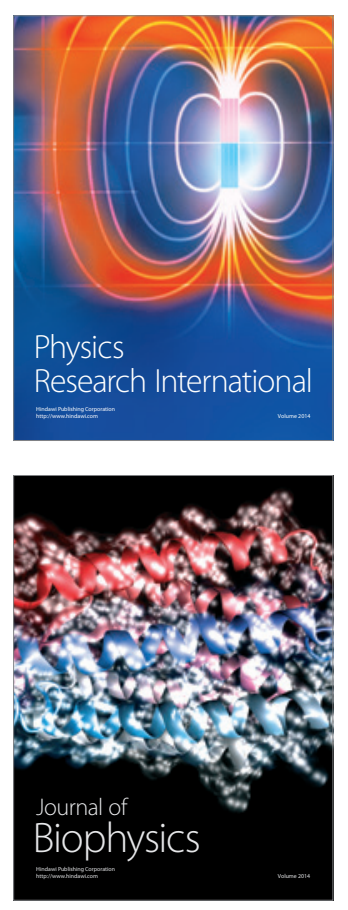
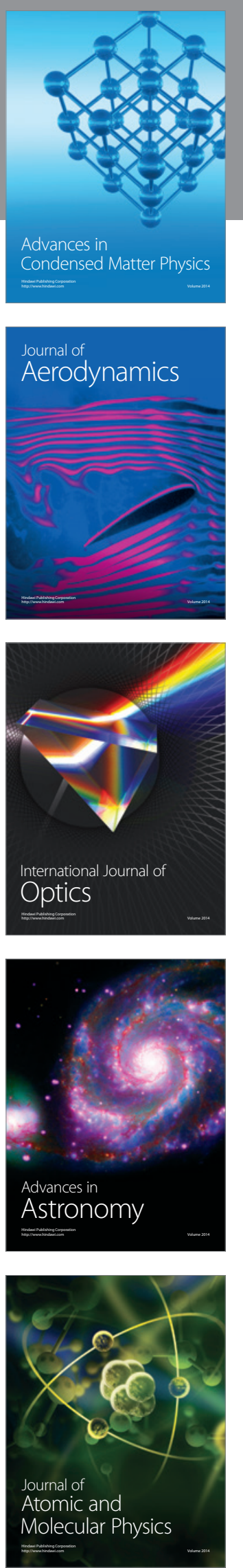